\title{
DENTAL APPLICATIONS OF HYDROXYAPATITE CEMENT - A PROMISING CHALLENGE
}

\author{
Milkov M. \\ Department of Material Science, Faculty of Dental Medicine, Medical University of Varna
}

Reviewed by: assoc. prof. Pl. Nedev

\begin{abstract}
Recently, hydroxyapatite cement is widely used in restorative otologic and craniofacial surgery as well as in dental implantology. The present survey illustrates the recent achievements in the characterization of the main clinical properties of this specific prosthetic material such as flexural strength, porosity, crystallinity, density, live cell density and viability, etc. A special attention is devoted to the advantages of the porous, sintered and nanocrystalline hydroxyapatite cements as grafting materials.
\end{abstract}

Key words: hydroxyapatite cement, properties, ossiculoplasty, dental implants, nanotechnology

\section{Hydroxyapatite usage in otologic surgery}

Hydroxyapatite cement enjoys a rising successful application in otology predominantly for ossiculoplasty in cases of severe hearing impairment and hearing loss. Bone cements have been used as prosthetic material in craniofacial surgery, and their adhesive and osseointegrative properties make them ideal for use in ossicular reconstruction (5). Hydroxyapatite mechanically bonds the ossicles at the time of application resulting in osseointegration with ossicular bone. Histologically, there is little evidence of inflammation or foreign body reaction. Such prostheses present with excellent biocompatibility (8). The biocompatibility of hydroxylapatite-polyethylene composite implants (HAPEX, Smith and Nephew) is examined by light, transmission and scanning electron microscopy (10). There is no accumulation of macrophages and foreign body giant cells due to a foreign body reaction. These composite implants are very suitable for reconstructive middle ear surgery.

Stapedectomy and total ossiculoplasty with cartilage-connecting hydroxyapatite prosthesis (Apaceram T-7 type) is effective and safe for stape fixation accompanied by fixation or erosion of the malleus and/or incus (7). Partial and total ossicular reconstruction prosthesis entirely made of dense hydroxylapatite yield high rates of air-bone gap reduction and high anatomic stability (13). The advantages of using an easily malleable, rapidly setting and rapidly hardening such as Mimix hydroxyapatite bone cement in reconstructing the ossicular chain abnormalities are proved by examination of preoperative and postoperative audiograms for 4-tone pure tone average, speech reception thresholds,

Address for correspondence:

M. Milkov, Dept. of Material Science, Faculty of Dental Medicine,

Medical University of Varna, 55 Marin Drinov Street,

BG-9002 Varna, Bulgaria

e-mail: mario.milkov@gmail.com word recognition scores, and air-bone gaps (3). Hydroxyapatite represents an excellent adjunct or alternative to ossiculoplasty with preformed prostheses.

\section{Properties of hydroxyapatite cement}

The effect of a bonding agent on in vitro biochemical activities of remineralizing resin-based calcium phosphate cements is analyzed (2). The ion solution concentrations show supersaturation for fluorapatite and hydroxyapatite suggesting a positive remineralization potential.

The crystallinity of the dental hydroxyapatite decreases with the age (9). Thermogravimetric measurements demonstrate a carbonate content increase with advancing age. FTIR spectroscopy reveals both B and A-type carbonate substitutions with the B-type greater than the A-type substitution by a factor up to $\sim 5$. Such an increase as a function of age can be deduced from the ratio of the $\mathrm{nu}_{2} \mathrm{CO}_{3}$ to the $\mathrm{nu}_{1} \mathrm{PO}_{4} \mathrm{IR}$ modes.

\section{Characteristics of porous hydroxyapatite}

Porous hydroxyapatite foam permits three-dimensional structure with fully interconnecting pores as well as excellent tissue response and good osteoconductivity. It is a good candidate as scaffold material for bone regeneration and as a synthetic bone substitute material. The effects of sintering temperature on the physical and compositional properties of this foam are evaluated by employing high sintering temperature starting at $1,300^{\circ} \mathrm{C}$ up to $1,550^{\circ} \mathrm{C}$ (11). Alpha tricalcium phosphate is formed and this hydroxyapatite foam becomes biphasic calcium phosphate. The latter shows higher osteoconductivity than hydroxyapatite alone. The sintering temperature of $1,550^{\circ} \mathrm{C}$ is recommended since this condition provides the most desirable physical properties of the biphasic calcium phosphate composition. 
The chemical interaction of 4-methacryloxyethyl trimellitic acid (4-MET) with hydroxyapatite using x-ray photoelectron spectroscopy (XPS) has been characterized (12). The peak representing COO- of 4-MET shifts to a lower binding energy when 4-MET is adsorbed onto hydroxyapatite. Deconvolution of this shifted peak discloses two components with a peak representing unreacted carboxyl groups and ester groups and a peak suggesting chemical bonding of other carboxyl groups to calcium of hydroxyapatite. Its XPS spectra treated with 4-MET also disclose the surface to be enriched in calcium and decreased in phosphorus indicating that phosphorus is extracted at a relatively higher rate than calcium. There is true chemical bonding of 4-MET with calcium present in hydroxyapatite.

\section{Characteristics of sintered hydroxyapatite}

The fatigue crack growth in human enamel is compared to that of sintered hydroxyapatite with similar crystallinity, chemistry and density (1). The fatigue crack growth exponent $(\mathrm{m})$ for enamel $(\mathrm{m}=7,7 \pm 1,0)$ is similar to that for hydroxyapatite $(m=7,9 \pm 1,4)$, whereas the crack growth coefficient $(\mathrm{C})$ for enamel $(\mathrm{C}=8,7 \mathrm{E}-04$ (mm/cycle) is significantly lower $(\mathrm{p}<0,0001)$ than that for hydroxyapatite $(\mathrm{C}=2,0 \mathrm{E}+00$ ( $\mathrm{mm} /$ cycle). Crack growth in the enamel occurs primarily along the prism boundaries and in regions of decussation, the microstructure promotes microcracking, crack bridging, deflection, and bifurcation. These mechanisms increase the crack growth resistance and result in a sensitivity to crack growth similar to bone and lower than that of human dentin but are not observed in the crack growth response of the sintered hydroxyapatite.

The layered structure of calcium phosphate cement (CPC) is developed by combining a macroporous CPC layer with a strong one (16). The flexural strength of CPC-scaffold decreases from $9,7 \pm 1,2$ to $1,8 \pm 0,3 \mathrm{MPa}(\mathrm{p}<0,05)$ when the porosity increases from $44,6 \%$ to $66,2 \%$. With a strong-layer reinforcement, however, this strength increases to $25,2 \pm 6,7$ and $100 \pm 14 \mathrm{MPa}$, respectively. These strengths match/exceed those of sintered porous hydroxyapatite implants and cancellous bone. Nano-sized hydroxyapatite crystals can form the scaffold matrix of CPC with chitosan. Such an in situ-hardening nano-apatite is useful in moderate stress-bearing applications, with macroporosity to enhance tissue in-growth and implant resorption.

\section{Usage of nanocrystalline hydroxyapatite in dentistry}

The proliferation of human periodontal ligament cells cultured in the presence of nano-hydroxyapatite paste and enamel matrix derivative is determined by incorporation of bromodeoxyuridine in the DNA of proliferating cells (6). Nanocrystalline hydroxyapatite shows two-fold less proliferation potential than enamel matrix derivative, but both substrates increase the proliferation rate significantly $(p<0,05)$ than the negative control. This is mechanistically linked to activation of the epidermal growth factor receptor. Nanocrystalline hydroxyapatite paste is a stimulator of cell proliferation, possibly contributing to the main processes of periodontal tissue regeneration.

This material (100\% Nanobone, Artoss, Rostock, Germany) embedded in a silica matrix is used as grafting material by the lateral lift technique (4). Tiolox/Tiologic implants (Dentaurum, Ispringen, Germany) are simultaneously placed, and after 6 to 9 months 37 implants are restored with fixed dental prostheses. The peri-implant parameters, periotest measurements and the restorations are evaluated. The implant success rate is $100 \%$ after 3 years. The periotest values (between -7 and -6) after implant abutment connection indicate a solid osseointegration. The mean rates of the marginal bone loss over the first year are higher (mesial: $-0,55$; distal: $-0,51 \mathrm{~mm}$ ) than the annual rates thereafter (mesial: $-0,09 \mathrm{~mm}$; distal: $-0,08 \mathrm{~mm}$ ). The prosthodontic and esthetic evaluation reveals a successful outcome.

Although CPC is a promising material for dental, periodontal, and craniofacial repairs, its use requires on-site powder-liquid mixing that increases the surgical placement time and raises concerns of insufficient and inhomogeneous mixing. A formulation of premixed CPC (PCPC) with rapid setting, high strength, and good in vitro cell viability is determined from CPC powder+non-aqueous liquid + gelling agent + hardening accelerator (15). Five PCPCs are developed: PCPC-tartaric, PCPC-malonic, PCPC-citric, PCPC-glycolic, and PCPC-malic. They are compared for setting time, diametral tensile strength, and osteoblast cell compatibility. Setting time for PCPC-tartaric is $8,2 \pm 0,8 \mathrm{~min}$, i. e., significantly less than the $61,7 \pm 1,5 \mathrm{~min}$ of the premixed control $(p<0,01)$. On the $7^{\text {th }}$ day of immersion, the diametral tensile strength of PCPC-tartaric reaches $6,5 \pm 0,8 \mathrm{MPa}$, i. e., higher than $4,5 \pm 0,8 \mathrm{MPa}$ of the premixed control $(\mathrm{p}=0,036)$. Osteoblast cells display a polygonal morphology and attach to the nano-hydroxyapatite crystals in the PCPCs. All the new cements have similar live cell density values $(p=0,126)$ indicating that the new PCPCs are as cell compatible as the non-premixed biocompatible CPC control. Their cell viability does not differ from that of this control. PCPCs eliminate the powder-liquid mixing during surgery and improve the cement performance. They support cell attachment and yield a high cell density and viability. Their mechanical strengths reaches the strengths of sintered porous hydroxyapatite implants and cancellous bone.

A nonrigid, durable CPC developed for periodontal bone repair (14) should provide the compliance for tooth mobility without fracturing the implant. Tetracalcium phosphate, dicalcium phosphate anhydrous and biopolymer chitosan are used. Specimens are characterized by a flexural test, scanning electron microscopy and powder x-ray diffraction. After 28 days of immersion, the new cement has a flexural strength of 5,2 $\pm 1,0 \mathrm{MPa}$ higher than $1,8 \pm 1,5 \mathrm{MPa}$ of the control $(p<0,05)$ and overlapping the strengths of sintered hydroxyapatite implants and cancellous bone. There is a high ductility with a strain at peak load of $6,5 \pm 1,3 \%$ as compared with $4,4 \pm 1,9 \%$ of the control. Both are 20 -fold higher than the $0,2 \%$ of the conventional CPC. 
Nano-sized hydroxyapatite crystals, similar to those in teeth and bones, are formed in these cements. The new self-hardening, nonrigid composite containing nano-hydroxyapatite crystals possess superior fracture resistance and are suitable for the regeneration of periodontal osseous defects.

\section{CONCLUSION}

Undoubtedly, continuous advances of nanotechnology promote a substantial quality improvement in a variety of medical and dental applications. Nanocrystalline hydroxyapatite cement represents a promising class of bone graft substitute in restorative dentistry.

\section{REFERENCES}

1. Bajaj D, Nazari A, Eidelman N, Arola DD. A comparison of fatigue crack growth in human enamel and hydroxyapatite.- Biomaterials, 29, 2008, 36, 4847-4854.

2. Dickens SH, Flaim GM. Effect of a bonding agent on in vitro biochemical activities of remineralizing resin-based calcium phosphate cements.- Dent. Mater., 24, 2008, 9, 1273-1280.

3. Goebel JA, Jacob A. Use of Mimix hydroxyapatite bone cement for difficult ossicular reconstruction.- Otolaryngol. Head Neck Surg., 132, 2005, 5, 727-734.

4. Heinemann F, Mundt T, Biffar R, Gedrange $\mathrm{T}$, Goetz W. A 3-year clinical and radiographic study of implants placed simultaneously with maxillary sinus floor augmentations using a new nanocrystaline hydroxyapatite.- $J$. Physiol. Pharmacol., 60, 2009, Suppl. 8, 91-97.

5. Hoffmann KK, Kuhn JJ, Strasnick B. Bone cements as adjuvant techniques for ossicular chain reconstruction.- Otol. Neurotol., 24, 2003, 1, 24-28.

6. Kasaj A, Willershausen B, Reichert C, Röhrig B, Smeets R, Schmidt M. Ability of nanocrystalline hydroxyapatite paste to promote human periodontal ligament cell proliferation.- J. Oral Sci., 50, 2008, 3, 279-285.
7. Kawano H, Matsuda K, Nakanishi H, Toyama K, Nagai N, Tono T. Ossiculoplasty with a cartilage-connecting hydroxyapatite prosthesis for tympanosclerotic stapes fixation.- Eur. Arch. Otorhinolaryngol., 267, 2010, 6, 875-879.

8. Kobayashi T, Gyo K, Shinohara T, Yanagihara N. Ossicular reconstruction using hydroxyapatite prostheses with interposed cartilage.Am. J. Otolaryngol., 23, 2002, 4, 222-227.

9. Leventouri T, Antonakos A, Kyriacou A, Venturelli R, Liarokapis E, Perdikatsis V. Crystal structure studies of human dental apatite as a function of age.- Int. J. Biomater., 2009, No 698547.

10. Meijer AG, Segenhout HM, Albers FW, van de Want HJ. Histopathology of biocompatible hydroxylapatite-polyethylene composite in ossiculoplasty.- ORL J. Otorhinolaryngol. Relat. Spec., 64, 2002, 3, 173-179.

11. Munar ML, Udoh K, Ishikawa K, Matsuya $\mathrm{S}, \mathrm{Nakagawa} \mathrm{M}$. Effects of sintering temperature over 1,300 degrees $\mathrm{C}$ on the physical and compositional properties of porous hydroxyapatite foam.- Dent. Mater. J., 25, 2006, 1, 51-58.

12. Nagakane K, Yoshida Y, Hirata I, Fukuda R, Nakayama Y, Shirai K, et al. Analysis of chemical interaction of 4-MET with hydroxyapatite using XPS.- Dent. Mater. J., 25, 2006, 4, 645-649.

13. Rondini-Gilli E, Grayeli AB, Borges Crosara PF, El Garem H, Mosnier I, Bouccara D, et al. Ossiculoplasty with total hydroxylapatite prostheses anatomical and functional outcomes.- Otol. Neurotol., 24, 2003, 4, 543-547.

14. Xu HH, Takagi S, Sun L, Hussain L, Chow LC, Guthrie WF, et al. Development of a nonrigid, durable calcium phosphate cement for use in periodontal bone repair.- J. Am. Dent. Assoc., 137, 2006, 8, 1131-1138.

15. Xu HH, Carey LE, Simon CG Jr, Takagi S, Chow LC. Premixed calcium phosphate cements: synthesis, physical properties, and cell cytotoxicity.Dent. Mater., 23, 2007a, 4, 433-441.

16. Xu HH, Burguera EF, Carey LE. Strong, macroporous, and in situ-setting calcium phosphate cement-layered structures.- Biomaterials, 28, 2007, 26, 3786-3796. 